\title{
The Immune Response in Nonmetastatic Axillary Lymph Nodes Is Associated with the Presence of Axillary Metastasis and Breast Cancer Patient Outcome
}

Carlos López, ${ }^{\star \dagger}$ Ramon Bosch, * Guifre Orero, * Anna Korzynska, ${ }^{\ddagger}$ Marcial García-Rojo, ${ }^{\S}$ Gloria Bueno, " María del Milagro Fernández-Carrobles, " Albert Gibert-Ramos, ${ }^{*}$ Lukasz Roszkowiak, ${ }^{\ddagger}$ Cristina Callau, * Laia Fontoura, * Maria-Teresa Salvadó, *† Tomás Álvaro, * Joaquín Jaén, * Albert Roso-Llorach," Montserrat Llobera, ** Julia Gil, ${ }^{\dagger \dagger}$

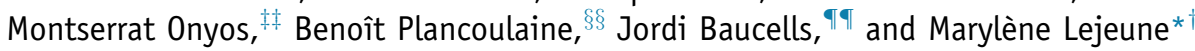

From the Departments of Pathology* and Oncology** and the Informatics Department, ${ }^{\mathbf{\top}}{ }^{\text {I }}$ Hospital de Tortosa Verge de la Cinta, Catalan Institute of Health, Pere Virgili Institute, Tortosa, Spain; the Nursing Department, ${ }^{\dagger}$ Campus Terres de l'Ebre, Universitat Rovira $i$ Virgili, Tortosa, Spain; the Laboratory of Processing and Analysis of Microscopic Images, ${ }^{\ddagger}$ Nalecz Institute of Biocybernetics and Biomedical Engineering, Polish Academy of Sciences (IBIB PAN), Warsaw, Poland; the Department of Pathology, ${ }^{\S}$ Hospital Universitario Puerta del Mar, Cádiz, Spain; the VISILAB, ${ }^{\mathbb{1}}$ Universidad de Castilla-La Mancha, Ciudad Real, Spain; the Institut Universitari d'Investigació en Atenció Primària Jordi Gol, ${ }^{\|}$Barcelona, Spain; the Department of Surgery, ${ }^{\dagger \dagger}$ Hospital

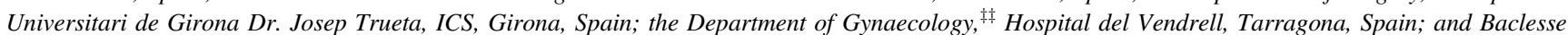
Center, ${ }^{\$ \S}$ Normandy University, Unicaen, Inserm, Interdisciplinary Research Unit for Cancer Prevention and Treatment, Caen, France

Accepted for publication November 12, 2019.

Address correspondence to Carlos López, Ph.D., or Marylène Lejeune, Ph.D, Molecular Biology and Research Section, Pathology Department, Hospital de Tortosa Verge de la Cinta, C/Esplanetes $\mathrm{n}^{\circ} 14,43500$-Tortosa, Spain. E-mail: clopezp.ebre. ics@gencat.cat or mlejeune. ebre.ics@gencat.cat.

\begin{abstract}
Tumor cells can modify the immune response in primary tumors and in the axillary lymph nodes with metastasis $\left(\mathrm{ALN}^{+}\right)$in breast cancer $(\mathrm{BC})$, influencing patient outcome. We investigated whether patterns of immune cells in the primary tumor and in the axillary lymph nodes without metastasis ( $\mathrm{ALN}^{-}$) differed between patients diagnosed without $\mathrm{ALN}^{+}$(diagnosed-ALN ${ }^{-}$) and with $\mathrm{ALN}^{+}$(diagnosed$\mathrm{ALN}^{+}$) and the implications for clinical outcome. Eleven immune markers were studied using immunohistochemistry, tissue microarray, and digital image analysis in $141 \mathrm{BC}$ patient samples (75 diagnosed-ALN ${ }^{+}$and 66 diagnosed-ALN ${ }^{-}$). Two logistic regression models were derived to identify the clinical, pathologic, and immunologic variables associated with the presence of $\mathrm{ALN}^{+}$at diagnosis. There are immune patterns in the $\mathrm{ALN}^{-}$associated with the presence of $\mathrm{ALN}^{+}$at diagnosis. The regression models revealed a small subgroup of diagnosed-ALN ${ }^{+}$with $\mathrm{ALN}^{-}$immune patterns that were more similar to those of the $\mathrm{ALN}^{-}$of the diagnosed-ALN ${ }^{-}$. This small subgroup also showed similar clinical behavior to that of the diagnosed-ALN ${ }^{-}$. Another small subgroup of diagnosed-ALN ${ }^{-}$with $\mathrm{ALN}^{-}$ immune patterns was found whose members were more similar to those of the $\mathrm{ALN}^{-}$of the diagnosed$\mathrm{ALN}^{+}$. This small subgroup had similar clinical behavior to the diagnosed-ALN ${ }^{+}$. These data suggest that the immune response present in $\mathrm{ALN}^{-}$at diagnosis could influence the clinical outcome of $\mathrm{BC}$ patients. (Am J Pathol 2020, 190: 660-673; https://doi.org/10.1016/j.ajpath.2019.11.002)
\end{abstract}

Supported by Institute of Health Carlos III projects PI1 1/0488 (principal investigator: C.L.) and PI13/02501 (principal investigator: M.L.), cofunded with the European Union European through the European Regional Development Fund; and European Comission project AIDPATH FP7PEOPLE 612471 (principal investigator: G.B.). The biopsies were selected from the Banc de Tumors of the Hospital de Tortosa Verge de la Cinta, member of the Xarxa de Bancs de Tumors de Catalunya, sponsored by the Oncology Master Plan for Catalonia.

C.L. and R.B. contributed equally to this work.

Disclosures: None declared. 
Annually, breast cancer (BC) is responsible for the deaths of $>520,000$ women throughout the world. ${ }^{1}$ The axillary lymph node (ALN) is the place where the BC most often first disseminates, and the presence of any ALN with metastasis at diagnosis $\left(\mathrm{ALN}^{+}\right)$in a patient suggests that the patient will have a worse prognosis than one with ALNs without metastasis $\left(\mathrm{ALN}^{-}\right){ }^{2}$ In fact, two-thirds of patients diagnosed with $\mathrm{ALN}^{+}$(diagnosed-ALN ${ }^{+}$) will develop distant metastasis, the leading cause of death in cancer patients, within 10 years of diagnosis. ${ }^{3}$

Lymph nodes are crucial centers for developing an effective immune response, and the study of the patient's lymph node immune status may be as important as the identification of aggressive tumor cell features. ${ }^{4}$ In fact, the immune response has shown itself to be directly involved in tumor progression. ${ }^{5,6} \mathrm{In} \mathrm{BC}$, the tissue immune response has an important role in tumor progression, the pathologic complete response to neoadjuvant therapy, and the relapse and survival of patients. ${ }^{7}$

Although most studies of BC patients have focused on evaluating the immune response context in the primary tumor, some have also addressed the immune context in ALN, most of them reporting negative effects on the immune response of the ALN arising from the presence of metastasis. Some studies have reported that nonmetastatic sentinel lymph nodes are more immunologically competent than those with metastasis, ${ }^{8,9}$ and two studies have described an immunosuppressive pattern in $\mathrm{ALN}^{+}$, principally due to an increase in the frequency of tumorassociated immature dendritic cells (DCs). ${ }^{9,10}$

There is substantial evidence that the tumor can also modify the immune context in $\mathrm{ALN}^{+}$as it does in the primary tumor. Nevertheless, to our knowledge, there is little evidence to indicate whether the tumor present in the $\mathrm{ALN}^{+}$ can modify the immune response in neighboring $\mathrm{ALN}^{-}$, or if these modifications in the $\mathrm{ALN}^{-}$might have repercussions on patient outcome. We only found one published study that demonstrates that the immune cells in $\mathrm{ALN}^{-}$from diagnosed-ALN ${ }^{+}$have different gene expression associated with the immune response compared with those from $\mathrm{ALN}^{-}$from patients diagnosed without $\mathrm{ALN}^{+}$ (diagnosed-ALN ${ }^{-}$). ${ }^{11}$ In the present study, we investigated whether the in situ patterns of immune cell populations present in the $\mathrm{ALN}^{-}$differ between diagnosed-ALN ${ }^{+}$and diagnosed-ALN ${ }^{-}$and how their different immune composition might affect the clinical outcome of BC.

\section{Materials and Methods}

\section{Study Design and Participants}

This retrospective cohort study was performed on the biopsies of 141 patients diagnosed with invasive breast carcinoma of no special type, between 2000 and 2008, provided by the Tumor Bank of the Department of Pathology of the Hospital de Tortosa Verge de la Cinta (Tortosa,
Spain). Patients were divided into two groups: 75 diagnosed-ALN ${ }^{+}$and 66 diagnosed-ALN ${ }^{-}$. Metastases in the ALN had been identified histologically. Only 13 patients $\left(17.3 \%\right.$ of diagnosed-ALN $\left.{ }^{+}\right)$had micrometastases in their ALN; the other diagnosed-ALN ${ }^{+}$had metastasis or both micrometastases and metastases. The mean (SD) of affected ALN in the diagnosed-ALN ${ }^{+}$was 4.6 (6.2). Regarding the treatments administered to the patients, $100 \%$ of diagnosed$\mathrm{ALN}^{-}$and $96.8 \%$ of diagnosed-ALN ${ }^{+}$received adjuvant therapy (hormonal therapy and/or chemotherapy) and $84.6 \%$ of diagnosed-ALN ${ }^{-}$and $79.7 \%$ diagnosed-ALN ${ }^{+}$received radiotherapy. All patients included in the study had biopsies with enough representative tissue from the primary tumor and from the $\mathrm{ALN}^{-}$paraffin blocks to perform the proposed analyses. The $\mathrm{ALN}^{-}$cases chosen for the study were not sentinel lymph nodes, as differences have previously been observed in the immune composition of sentinel lymph nodes compared with non-sentinel lymph nodes of diagnosed-ALN ${ }^{-} .^{10,12}$ The study was approved by the Ethics Committee of the Hospital Joan XXIII de Tarragona (reference 22p/2011), and patients provided their written informed consent, in accordance with Spanish law. The Strengthening the Reporting of Observational Studies in Epidemiology guidelines were followed.

\section{Procedures}

The immune populations were evaluated in two representative tissue cylinders of 2-mm diameter taken from paraffin-embedded blocks of the surgical biopsies of each patient. Two pathologists (R.B. and J.J) selected four representative areas from new hematoxylin-eosin slides: two representative areas from the primary tumor biopsies with enough tumoral tissue to find immune infiltrates and two areas from the central region of the $\mathrm{ALN}^{-}$biopsies. The morphology of the ALN is heterogeneous in human breast cancer; therefore, the same region from which to extract the tissue cylinders was always chosen. A total of 564 cylinders were obtained [(141 patients $\times 2$ cylinders/region $) \times(2$ intratumoral regions +2 different $\left.\mathrm{ALN}^{-}\right)$] and incorporated into tissue microarrays (TMAs), as previously described. ${ }^{13}$ TMA technology is an invaluable technique for high throughput of large numbers of cases; and although the level of correlation between TMAs and whole tissue sections may not be considered ideal at the diagnostic level for individual patients, in the research setting of large numbers of samples in clinical trials or research studies, this is widely regarded as adequate. ${ }^{14}$ In fact, a search of PubMed with the terms tissue microarray breast cancer immune yielded $>100$ articles, highlighting the wide use of TMAs in the evaluations of tumor microenvironment biomarkers and immune checkpoint molecules. ${ }^{15,16}$ The combined use of image analysis procedures and TMAs allows a completely automated analysis. It proved to be an emerging and an extremely important method in biomarker research to fast 
track discovery streamline workflow and produce objective, reproducible biomarker data. ${ }^{17}$

Each TMA block contained approximately 50 cylinders, giving 12 TMAs. Eleven slides were sectioned from each TMA to stain the 11 immune markers studied. The immune selection of the markers for the study was based on the results of previous works that have demonstrated that the different types of lymphocytes, the macrophages, and the DCs are associated with BC patients' outcome. ${ }^{18-20}$

Immunohistochemical detection of the immune cells was performed on each slide using the appropriate primary antibody (Figure 1): T-helper lymphocytes (anti-CD4; clone 4B12; Dako, Santa Clara, CA), cytotoxic T lymphocytes (anti-CD8; clone C8/144B; Dako), natural killer cells (antiCD57; clone NK1; Zymed, ThermoFisher Scientific, Waltham, MA), regulatory $\mathrm{T}$ cells [anti-forkhead box P3 (antiFOXP3); clone 236A/E7; CNIO, Madrid, Spain], macrophages (anti-CD68; clone KP1; Dako), follicular DC (antiCD21; clone 1F8; Dako), Langerhans DC (anti-CD1a; clone 010; Dako), plasmacytoid DC (anti-CD123; clone 6H6; eBioscience, San Diego, CA), interdigitant DC (anti-S100; polyclonal; Leica Microsystems GmbH, Wetzlar, Germany), lysosomal associated membrane protein 3 (LAMP3 DC; anti-CD208; polyclonal; Proteintech, Rosemont, IL), and mature DC (anti-CD83; clone1H4b; Leica Microsystems $\mathrm{GmbH}$, Wetzlar, Germany). Final detection using the ENDVISION FLEX method (Dako), using the chromogen diaminobenzidine as a substrate and counterstained with hematoxylin, was performed in accordance with the supplier's instructions and laboratory protocol.

The 132 stained slides (12 TMAs $\times 11$ immune markers) were scanned using the Aperio ScanScope XT scanner (Leica Microsystems $\mathrm{GmbH}$, Nussloch, Germany) at $\times 40$ magnification to provide digital images of the slides in Tagged Image File Format (TIFF) at a resolution of 0.25 $\mu \mathrm{m} /$ pixel and with a mean size of approximately $25 \mathrm{~GB}$. Their production involves whole slide imaging, the most recent digital imaging modality employed by pathology departments worldwide. ${ }^{21}$ Because of the difficulties of automatically analyzing these big digital images, each cylinder of the original whole slide image from each TMA was extracted as a single image using an automatic tool previously developed by members of our team. ${ }^{22,23}$ The 6204 extracted images (564 cylinders $\times 11$ immune markers) were stored in TIFF format and classified according to the stained immune marker and its case number. Images of each cylinder were approximately $500 \mathrm{MB}$ in size.

Digital image analysis is currently used in breast cancer, ${ }^{24,25}$ but it could also be applied to the study of tumor microenvironment elements. ${ }^{26}$ The tissue cylinder areas and immune markers were evaluated by digital image analytical procedures that had been developed and tested for their ability to quantify immunohistochemical markers in cancer. $^{13,25,27,28}$ The concentration of immune populations in each cylinder was calculated as the percentage of positively stained areas of each immune marker relative to the whole
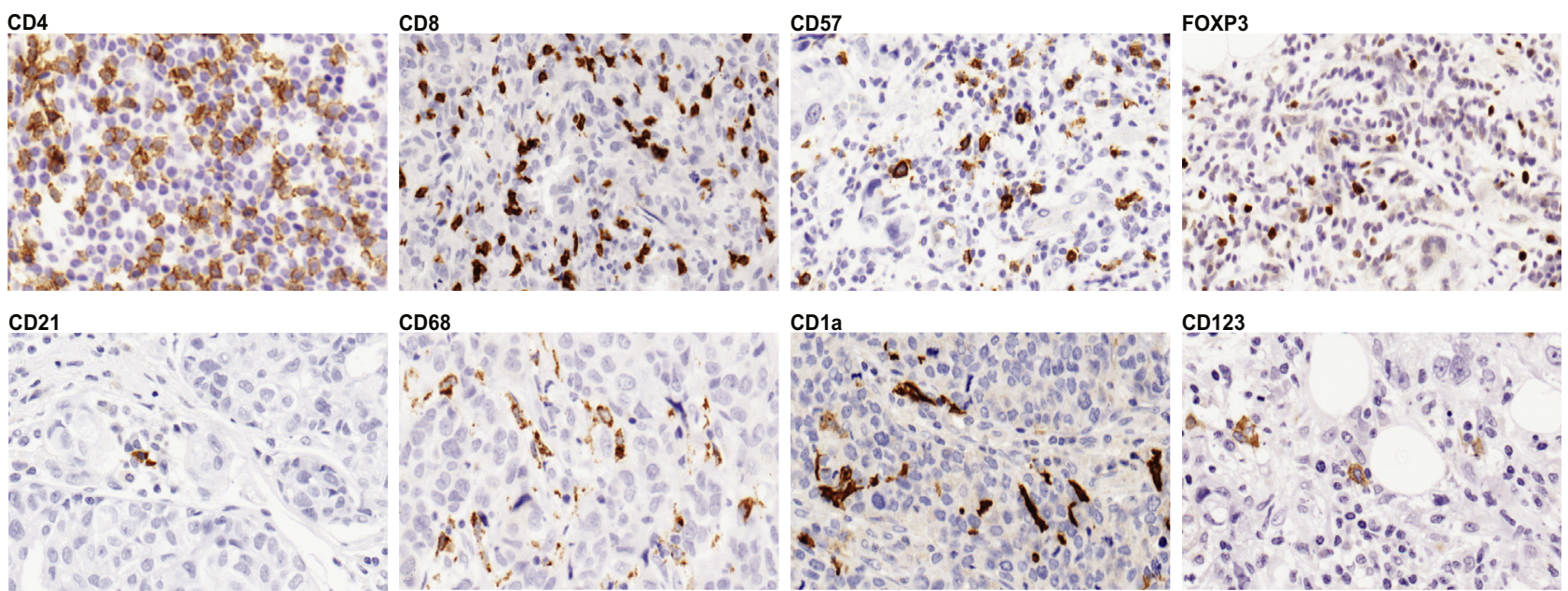

CD68
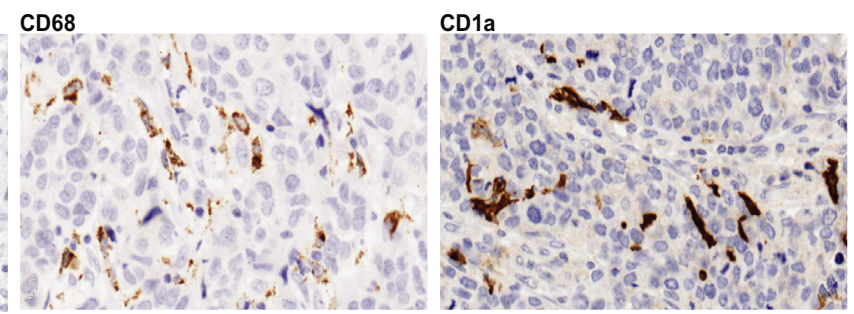

CD123
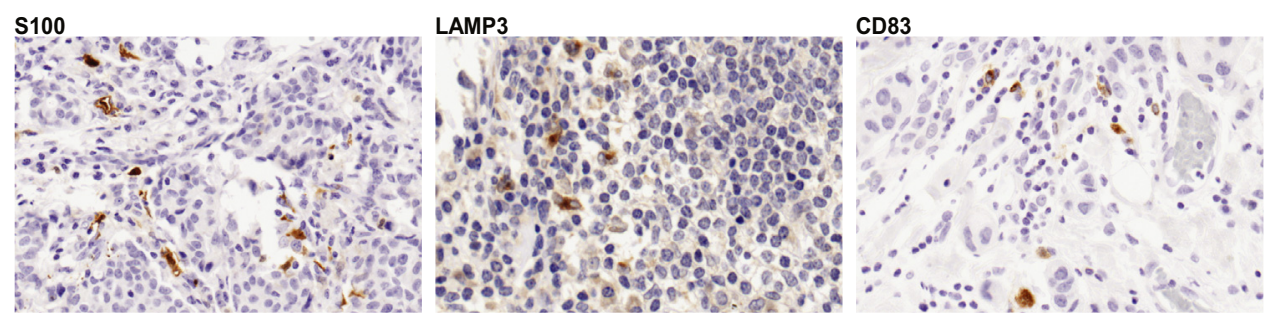

Figure 1 Immunohistochemical staining patterns for the studied immune markers in formalin-fixed, paraffin-embedded sections. Representative examples of membrane (CD4, CD8, and CD21), cytoplasmic [CD68, CD123, and lysosomal associated membrane protein 3 (LAMP3)], membrane and/or cytoplasmic (CD57, CD1a, and CD83), nuclear and/or cytoplasmic (S100), and nuclear [forkhead box P3 (F0XP3)] reactivity of the biomarkers. Original magnification, $\times 20$. 
area of the cylinder using the digital image analytical procedures. For each patient, the mean of the percentages of the two cylinders for each stained marker was used. In cases where one of the cylinders was lost because of a bad stain, loss of tissue, or bad image quality, the information from a single cylinder was used.

Values of the following clinical and pathologic variables were collected: age, tumor diameter, lymphovascular invasion (LVI), perineural invasion (PNI), histologic grade, estrogen receptor status, progesterone receptor status, HER2 status, proliferation index (Ki-67), menopausal status, and molecular profile. Overall survival (OS), cancer-specific survival (CSS), and time to progression (TTP) after 10 years of follow-up were also evaluated. OS was defined as the time from diagnosis to the end of follow-up, or to death from any cause; CSS was defined as the time from diagnosis to the end of the follow-up, or death due to BC; TTP was defined as the time from the date of first diagnosis until the end of the follow-up, or until the disease started to spread to other parts of the body.

\section{Statistical Analysis}

The differences in the concentrations of the studied immune populations evaluated in the primary tumor and in the $\mathrm{ALN}^{-}$ were evaluated using $t$-tests or $U$-tests, as appropriate. The same tests were used to evaluate the differences in the quantitative clinical and pathologic variables (age and tumor diameter). The $\chi^{2}$ test or the Fisher exact test was used to identify differences in the frequencies of the categorical clinical and pathologic variables [LVI, PNI, histologic grade, estrogen receptor status, progesterone receptor status, HER2 amplification, proliferation index (Ki-67), menopausal status, and molecular profile] between the two groups of patients.

Univariate logistic regressions were fitted for each variable to examine the association with having $\mathrm{ALN}^{+}$at diagnosis. Two multivariate logistic regression models were then developed to identify the variables independently associated with having or not having $\mathrm{ALN}^{+}$at diagnosis. Both models initially considered all the variables that had a significance of $P<0.1$ in the univariate analyses. However, variables with a high odds ratio (OR) were not considered in the second model to determine whether they might be masking the effect of other variables that were also associated with the presence of $\mathrm{ALN}^{+}$at diagnosis. The goodness of fit of all the variables considered in the multivariate analyses was examined by the Hosmer-Lemeshow test. Regarding the sample size of the study, it has been suggested that the data should contain at least 10 events for each variable entered into a logistic regression model ${ }^{29}$ or even less in some cases. ${ }^{30}$ In the present study, 66 events and four variables were included in the final multivariate models, which constitutes a big enough sample size for the multivariate analysis. The models were validated using the bootstrapping simulation technique available in IBM SPSS Statistics 21.0 (IBM, Armonk, NY), with a 95\% CI. A total of 10,000 samples were used for the simulation.
The ability of these models to correctly assign patients to the diagnosed-ALN ${ }^{+}$or diagnosed-ALN ${ }^{-}$groups was evaluated using sensitivity, specificity, and receiveroperating characteristic curves. Statistically significant differences in the area under the curve (AUC) between the receiver-operating characteristic curves were identified using the test developed by DeLong et al. ${ }^{31}$

A comparison of the distribution of survival times (OS, CSS, and TTP) after a 10-year follow-up in the diagnosed$\mathrm{ALN}^{+}$and diagnosed-ALN ${ }^{-}$groups was estimated by the Kaplan-Meier method using the log-rank test. The survival times were also compared for patients predicted with the models to be with $\mathrm{ALN}^{+}$(predicted-ALN ${ }^{+}$) and those predicted with the models to be without $\mathrm{ALN}^{+}$(predicted$\mathrm{ALN}^{-}$) on the basis of various multivariate logistic regression models. Finally, for each model, the survival times were compared between those diagnosed-ALN ${ }^{+}$patients correctly classified as being predicted-ALN ${ }^{+}$and those incorrectly classified as being predicted-ALN ${ }^{-}$. The survival times of those patients correctly classified as being predicted-ALN ${ }^{-}$ and incorrectly classified as being predicted-ALN ${ }^{+}$were also compared in the diagnosed-ALN ${ }^{-}$group. $P<0.05$ was considered statistically significant in all analyses. All statistical analyses were performed with IBM SPSS Statistics 21.0 or STATA 14.0 (StataCorp LLC, College Station, TX).

\section{Results}

\section{Immune and Clinical Differences}

Table 1 shows the differences in the clinical and pathologic variables between the two groups of patients. Diagnosed$\mathrm{ALN}^{+}$had significantly larger tumors, higher percentages of LVI and PNI, and a higher histologic grade than diagnosed$\mathrm{ALN}^{-}$. The differences in the concentration of immune cell populations calculated with the digital image analysis procedures between the two groups of patients are summarized in Table 2. No significant differences in the primary tumor were found, except for the follicular CD21 DC, which was present at a higher concentration in diagnosed-ALN ${ }^{+}$. Conversely, several significant differences in the immune cell populations of the $\mathrm{ALN}^{-}$were detected between diagnosed-ALN ${ }^{+}$and diagnosed-ALN ${ }^{-}$.

\section{Logistic Regression Models}

Table 3 shows the results of the univariate analysis and the two multivariate models. Considering the immune variables, in the multivariate models, the Hosmer-Lemeshow test revealed a poor fit only for CD21 concentration (data not shown), and therefore, the variable was dichotomized as absent or present, rather than considered as a continuous variable. In the case of the clinical and pathologic variables, LVI is one of the factors most strongly associated with $\mathrm{ALN}^{+}$, and so was excluded from the second multivariate model to determine whether this variable masked the effect of others that might also be 
Table 1 Differences in the Clinical and Pathologic Variables of Diagnosed-ALN ${ }^{-}$and Diagnosed-ALN ${ }^{+}$

\begin{tabular}{|c|c|c|c|}
\hline Variable & $\begin{array}{l}\text { Diagnosed-ALN }{ }^{-} \\
(n=75)\end{array}$ & $\begin{array}{l}\text { Diagnosed-ALN }{ }^{+} \\
(n=66)\end{array}$ & $P$ value \\
\hline Age, years & $61.2(10.6)$ & $59.7(12.0)$ & $0.434^{*}$ \\
\hline $\begin{array}{l}\text { Tumor } \\
\quad \text { diameter, } \mathrm{mm}\end{array}$ & $15.0(13.0)$ & $22.0(13.0)$ & $<0.001^{\dagger}$ \\
\hline \multicolumn{4}{|l|}{ LVI } \\
\hline Yes & $8(12.1)$ & $57(77.0)$ & \multirow[t]{2}{*}{$<0.001^{\ddagger}$} \\
\hline No & $58(87.9)$ & $17(23.0)$ & \\
\hline \multicolumn{4}{|l|}{ PNI } \\
\hline Yes & $10(15.2)$ & $30(40.5)$ & \multirow[t]{2}{*}{0.001} \\
\hline No & $56(84.8)$ & $44(59.5)$ & \\
\hline \multicolumn{4}{|l|}{ Histologic grade } \\
\hline 1 & $22(33.3)$ & $8(10.7)$ & \multirow[t]{3}{*}{$0.002^{\ddagger}$} \\
\hline 2 & $28(42.4)$ & $34(45.3)$ & \\
\hline 3 & $16(24.3)$ & $33(44.0)$ & \\
\hline \multicolumn{4}{|l|}{ ER expression } \\
\hline Positive & $48(72.7)$ & $53(70.7)$ & \multirow[t]{2}{*}{$0.787^{\ddagger}$} \\
\hline Negative & $18(27.3)$ & $22(29.3)$ & \\
\hline \multicolumn{4}{|l|}{ PR expression } \\
\hline Positive & $43(65.2)$ & $41(54.7)$ & \multirow[t]{2}{*}{$0.206^{\ddagger}$} \\
\hline Negative & $23(34.8)$ & $34(45.3)$ & \\
\hline \multicolumn{4}{|l|}{ HER2 amplification } \\
\hline Amplified & $16(24.6)$ & $20(26.7)$ & \multirow[t]{2}{*}{$0.782^{\ddagger}$} \\
\hline Nonamplified & $49(75.4)$ & $55(73.3)$ & \\
\hline \multicolumn{4}{|l|}{ Ki-67 degree } \\
\hline Low & $23(34.9)$ & $16(21.9)$ & \multirow[t]{3}{*}{$0.184^{\ddagger}$} \\
\hline Medium & $22(33.3)$ & $25(34.3)$ & \\
\hline High & $21(31.8)$ & $32(43.8)$ & \\
\hline \multicolumn{4}{|l|}{ Menopausal status } \\
\hline Premenopausal & $6(9.8)$ & $15(22.1)$ & \multirow[t]{2}{*}{$0.060^{\ddagger}$} \\
\hline Post-menopausal & $55(90.2)$ & $53(77.9)$ & \\
\hline \multicolumn{4}{|l|}{ Molecular profile } \\
\hline Luminal A & $42(64.6)$ & $46(61.4)$ & \multirow[t]{4}{*}{$0.983^{\ddagger}$} \\
\hline Luminal B & $8(12.3)$ & $10(13.3)$ & \\
\hline HER2 & $8(12.3)$ & $10(13.3)$ & \\
\hline Triple negative & $7(10.8)$ & $9(12.0)$ & \\
\hline
\end{tabular}

${ }^{*} t$-test [data are given as means (SD)].

${ }^{\dagger} U$-test [data are given as median (interquartile range)].

${ }^{\ddagger} \chi^{2}$ or the Fisher exact test [data are given as $n(\%)$ ].

$E R$, estrogen receptor; $H E R$, human epidermal growth factor receptor; LVI, lymphovascular invasion; PNI, perineural invasion; PR, progesterone receptor.

associated with the presence of $\mathrm{ALN}^{+}$at diagnosis. Moreover, CD21 was also excluded from this second multivariate model because its absence produced a more parsimonious model. The ORs and CIs before removing CD21 DC in model 2 were as follows: tumor diameter (OR, 1.10; 95\% CI, 1.04-1.16; $P=0.001)$, the CD68 macrophages (OR, 1.25; 95\% CI, 1.07-1.46; $P=0.006$ ), DC population CD123 (OR, 0.47; 95\% CI, 0.29-0.76; $P=0.002$ ), and DC S100 in the $\mathrm{ALN}^{-}$ (OR, 1.24; 95\% CI, 1.04-1.47; $P=0.019$ ). The variables included in model 2 after removing CD21 did not change, and their OR and level of significance in the model were stable (Table 3).

The comparison of these two models allows us to check the association of the other variables with the $\mathrm{ALN}^{+}$and indicates that in the second model (without LVI and CD21) the concentrations of S100 (risk factor) and CD123 (protective factor) immune cell populations in $\mathrm{ALN}^{-}$were new variables associated with the presence of $\mathrm{ALN}^{+}$at diagnosis. Both models were validated using the bootstrapping simulation, after which it was observed that the same variables were present in the models. The results of validation for model 1 were as follows: tumor diameter (OR, 1.05; 95\% CI, 1.00-1.09; $P=0.039)$, LVI (OR, 71.03; 95\% CI, 16.42-307.32; $P<0.001)$, intratumoral DC population CD21 (OR, 7.59; 95\% CI, 1.97-29.16; $P=0.003$ ), and the CD68 macrophages in the $\mathrm{ALN}^{-}(\mathrm{OR}, 1.22 ; 95 \% \mathrm{CI}$, $1.06-1.40 ; P=0.005)$. In this validation, most of the results remained almost the same except for the LVI that exhibited a considerable reduction in its OR. The results of the validation of model 2 were as follows: tumor diameter (OR, 1.08; 95\% CI, 1.03-1.14; $P=0.001)$, CD68 macrophages (OR, 1.27; 95\% CI, 1.11-1.44; $P<0.001)$, DC population CD123 (OR, 0.45; 95\% CI, 0.29-0.71; $P=0.001)$, and DC S100 in the $\mathrm{ALN}^{-}(\mathrm{OR}, 1.25 ; 95 \% \mathrm{CI}$,

Table 2 Comparison of Percentages of Immune Populations in the Primary Tumor and $\mathrm{ALN}^{-}$Regions between Diagnosed-ALN ${ }^{+}$ and Diagnosed-ALN

\begin{tabular}{lccr}
\hline $\begin{array}{l}\text { Primary tumor or } \\
\text { ALN }^{-} \text {region }\end{array}$ & $\begin{array}{l}\text { Diagnosed-ALN } \\
(n=75)\end{array}$ & $\begin{array}{l}\text { Diagnosed-ALN } \\
(n=66)\end{array}$ & $P$ value \\
\hline Primary tumor & & & \\
CD4 & $1.0(2.4)$ & $0.8(1.5)$ & $0.160^{*}$ \\
CD8 & $1.1(2.8)$ & $1.3(2.6)$ & $0.872^{*}$ \\
CD57 & $0.1(0.3)$ & $0.2(0.5)$ & $0.151^{*}$ \\
F0XP3 & $0.1(0.2)$ & $0.1(0.2)$ & $0.432^{*}$ \\
CD21 & $0.000(0.002)$ & $0.016(0.04)$ & $<0.001^{*}$ \\
CD68 & $2.8(3.6)$ & $2.8(2.3)$ & $0.748^{*}$ \\
CD1a & $0.1(0.3)$ & $0.1(0.2)$ & $0.315^{*}$ \\
CD123 & $0.00(0.04)$ & $0.00(0.03)$ & $0.975^{*}$ \\
S100 & $0.1(0.4)$ & $0.2(0.3)$ & $0.369^{*}$ \\
CD208 & $0.02(0.08)$ & $0.03(0.15)$ & $0.225^{*}$ \\
CD83 & $0.1(0.1)$ & $0.1(0.2)$ & $0.568^{*}$ \\
ALN ${ }^{-}$ & & & \\
CD4 & $56.9(12.5)$ & $54.0(13.6)$ & $0.218^{\dagger}$ \\
CD8 & $11.8(9.4)$ & $16.0(8.3)$ & $0.006^{*}$ \\
CD57 & $0.2(0.2)$ & $0.2(0.3)$ & $0.196^{*}$ \\
F0XP3 & $2.2(1.0)$ & $2.3(1.1)$ & $0.575^{\dagger}$ \\
CD21 & $0.8(1.8)$ & $0.7(1.4)$ & $0.250^{*}$ \\
CD68 & $7.4(5.0)$ & $9.6(5.7)$ & $0.006^{*}$ \\
CD1a & $1.5(2.7)$ & $1.1(3.2)$ & $0.911^{*}$ \\
CD123 & $1.7(2.1)$ & $1.3(1.6)$ & $0.015^{*}$ \\
S100 & $3.6(3.5)$ & $5.0(5.3)$ & $0.009^{*}$ \\
CD208 & $0.2(0.3)$ & $0.3(0.6)$ & $0.134^{*}$ \\
CD83 & $1.1(1.4)$ & $0.9(1.1)$ & $0.354^{*}$ \\
\hline
\end{tabular}

The values represented in the table for the different comparisons are the means (SD) or the median (interquartile range) of the percentage of positive area expressed for each of the studied markers at each of the two locations.

${ }^{*} U$-test [data are given as median (interquartile range)].

${ }^{\dagger} t$-test [data are given as means (SD)].

FOXP3, forkhead box P3. 
$1.07-1.46 ; P=0.006)$. In this second model, the OR of all variables remained almost the same.

\section{Predictive Models and Patient Assignment to Diagnosed Groups}

As mentioned in Materials and Methods, logistic regression models were used to assess the ability of the two models to correctly assign patients to the diagnosed-ALN ${ }^{+}$or diagnosed-ALN ${ }^{-}$groups. Under these conditions, diagnosed$\mathrm{ALN}^{+}$and diagnosed-ALN ${ }^{-}$were those patients determined by the pathologist to be with or without $\mathrm{ALN}^{+}$, respectively, whereas predicted-ALN ${ }^{-}$and predicted-ALN ${ }^{+}$ refer to those patients classified by the multivariate logistic regression model, depending on their clinical and pathologic variables and their immune-response patterns present in the $\mathrm{ALN}^{-}$. Incorrectly classified status was assigned to patients when their predicted ALN status (predicted-ALN ${ }^{+}$or predicted-ALN ${ }^{-}$) was not in accordance with their diagnosed ALN status (diagnosed-ALN ${ }^{+}$or diagnosed-ALN ${ }^{-}$).

At this point, the ability of these two models to correctly classify predicted-ALN ${ }^{+}$cases in the diagnosed-ALN ${ }^{+}$ group and predicted-ALN ${ }^{-}$cases in the diagnosed-ALN ${ }^{-}$ group was calculated using receiver-operating characteristic curves. Model 1 had a sensitivity of $83.9 \%$, a specificity of 90.0\%, and an AUC of 0.940 (95\% CI, 0.901-0.970), whereas model 2 had a sensitivity of $71.4 \%$, a specificity of $87.1 \%$, and an AUC of 0.840 (95\% CI, 0.773-0.908) (Supplemental Figure S1). The difference in the AUC between the two versions of model 2 (with or without CD21) was small (1.9\%), and the statistical test of DeLong et $\mathrm{al}^{31}$ indicated that the difference was not significant $(P=0.1)$, so the more parsimonious model 2 (without CD21) was considered subsequently, as previously mentioned. ${ }^{31}$ The significant difference between the AUCs revealed by the test of DeLong et $\mathrm{al}^{31}(P=0.01)$ indicated that model 1 was better than model 2 at correctly predicting patient ALN status.

\section{Predictive Models and Patient Outcome}

After noting the predictive capability of the models, the different survival times of diagnosed-ALN ${ }^{+}$and diagnosed-ALN ${ }^{-}$and those of the predicted-ALN ${ }^{+}$and predicted-ALN ${ }^{-}$for both models were compared (Figure 2). In all cases, diagnosed-ALN ${ }^{-}$and predicted-ALN ${ }^{-}$had better outcomes than diagnosed-ALN ${ }^{+}$and predicted$\mathrm{ALN}^{+}$, respectively. The differences between survival curves of the diagnosed-ALN ${ }^{+}$and diagnosed-ALN ${ }^{-}$ groups were $13.2 \%$ for OS, $8.6 \%$ for CSS, and $9.9 \%$ for TTP. The differences between survival curves of predicted-ALN ${ }^{+}$and predicted-ALN ${ }^{-}$were more pronounced for the predictions based on model 2 (26.4\% for OS, $19.8 \%$ for CSS, and $21.6 \%$ for TTP) than for those based on model 1 (20.6\% for OS, $15.8 \%$ for CSS, and $17.4 \%$ for TTP) (Figure 2). Thus, although model 1 was better at correctly predicting patient ALN status, model 2 was better at discriminating patient outcome.

To establish whether the clinical behavior differed between correctly and incorrectly predicted patients, their OS, CSS, and TTP curves were compared for model 1 (Figure 3) and model 2 (Figure 4). The upper row of both figures shows the diagnosed-ALN ${ }^{+}$that were correctly classified as predicted-ALN ${ }^{+}$and incorrectly classified as predicted$\mathrm{ALN}^{-}$. The lower rows show the diagnosed-ALN ${ }^{-}$that were incorrectly classified as predicted-ALN ${ }^{+}$and correctly classified as predicted-ALN ${ }^{-}$. In all the graphs of both figures, the survival curves of predicted patients were compared with the survival curves of diagnosed-ALN ${ }^{-}$and of the diagnosed-ALN ${ }^{+}$that are taken as the reference. These comparisons allowed us to confirm whether the predicted patients had similar clinical behavior to the group in which they were classified and different behavior to the opposite group, irrespective of whether the prediction was correct.

In model 1 (Figure 3), incorrectly predicted patients showed no statistically significant differences in any of the survival times when compared with diagnosed-ALN ${ }^{+}$or with diagnosed-ALN ${ }^{-}$. Moreover, incorrectly predicted$\mathrm{ALN}^{-}$showed no significant differences in any of the survival time measures compared with the correctly classified predicted-ALN ${ }^{+}$group $(P=0.132$ for OS, $P=0.115$ for CSS, and $P=0.100$ for TTP). Likewise, incorrectly predicted-ALN ${ }^{+}$were not significantly different from correctly predicted-ALN ${ }^{-}(P=0.120$ for OS, $P=0.062$ for CSS, and $P=0.057$ for TTP). These results indicate that incorrect classification had no effect on differential patient clinical outcome. On the other hand, as expected, correctly predicted-ALN ${ }^{+}$were significantly different from diagnosed-ALN ${ }^{-}$but not diagnosed-ALN ${ }^{+}$. The same effect was noted for the correctly classified predicted-ALN ${ }^{-}$, which showed significant differences from diagnosed$\mathrm{ALN}^{+}$, but not from diagnosed-ALN ${ }^{-}$.

In model 2 (Figure 4), the correctly predicted patients showed significantly different survival times from those of the opposite group of diagnosed patients (predicted-ALN ${ }^{+}$ versus diagnosed-ALN ${ }^{-}$and predicted-ALN ${ }^{-}$versus diagnosed-ALN ${ }^{+}$) and no differences with respect to the same group of diagnosed patients in which they were correctly classified (predicted-ALN ${ }^{+}$versus diagnosed-ALN ${ }^{+}$and predicted-ALN ${ }^{-}$versus diagnosed-ALN ${ }^{-}$). The main differences between model 2 and model 1 were that model 2 showed significant or nearly significant differences in the survival times between incorrectly predicted-ALN ${ }^{-}$and correctly classified predicted-ALN ${ }^{+}$and between incorrectly classified predicted-ALN ${ }^{+}$and correctly classified predicted-ALN ${ }^{-}$. The same was noted when comparing the incorrectly predicted-ALN ${ }^{-}$with the opposite group diagnosed-ALN ${ }^{+}$and comparing the incorrectly predicted$\mathrm{ALN}^{+}$with the diagnosed-ALN ${ }^{-}$. So, as illustrated in the upper row, although the great majority of diagnosed-ALN ${ }^{+}$ were correctly classified as predicted-ALN ${ }^{+}$, there was a 
Table 3 Univariate and Multivariate Logistic Regression Analysis of the Variables Associated with ALN ${ }^{+}$at Diagnosis

\begin{tabular}{|c|c|c|c|c|c|c|}
\hline Variable & $\begin{array}{l}\text { Univariate } \\
\text { OR }(95 \% \mathrm{CI})\end{array}$ & $P$ value & $\begin{array}{l}\text { Multivariate } 1 \\
\text { OR }(95 \% \mathrm{CI})\end{array}$ & $P$ value & Multivariate 2 OR (95\% CI) & $P$ value \\
\hline \multicolumn{7}{|c|}{ Clinical and pathologic variables } \\
\hline Tumor diameter, $\mathrm{mm}$ & $1.06(1.02-1.10)$ & 0.001 & $1.16(1.07-1.27)$ & $<0.001$ & $1.10(1.04-1.17)$ & 0.001 \\
\hline \multicolumn{7}{|l|}{ LVI } \\
\hline Present & $24.31(9.72-60.78)$ & $<0.001$ & $171.2(20.8-1411.2)$ & $<0.001$ & & \\
\hline Present & $3.82(1.69-8.65)$ & 0.001 & & & & \\
\hline Absent & 1.0 & & & & & \\
\hline \multicolumn{7}{|l|}{ Histologic grade } \\
\hline 3 & $5.67(2.08-15.50)$ & 0.001 & & & & \\
\hline 2 & $3.34(1.29-8.65)$ & 0.013 & & & & \\
\hline \multicolumn{7}{|l|}{ PR expression } \\
\hline Positive & $0.65(0.33-1.27)$ & 0.207 & & & & \\
\hline Negative & 1.0 & & & & & \\
\hline \multicolumn{7}{|l|}{ HER2 amplification } \\
\hline Amplified & $1.11(0.52-2.39)$ & 0.782 & & & & \\
\hline Nonamplified & 1.0 & & & & & \\
\hline \multicolumn{7}{|l|}{ PI (Ki-67) } \\
\hline High & $2.19(0.94-5.09)$ & 0.068 & & & & \\
\hline Medium & $1.63(0.69-3.85)$ & 0.262 & & & & \\
\hline Low & 1.0 & & & & & \\
\hline \multicolumn{7}{|l|}{ Menopausal status } \\
\hline CD4 & $0.97(0.87-1.08)$ & 0.558 & & & & \\
\hline CD8 & $0.99(0.90-1.10)$ & 0.900 & & & & \\
\hline CD57 & $1.02(0.97-1.08)$ & 0.403 & & & & \\
\hline F0XP3 & $1.00(0.99-1.00)$ & 0.160 & & & & \\
\hline \multicolumn{7}{|l|}{ CD21 } \\
\hline Presence & $5.35(2.54-11.30)$ & $<0.001$ & $9.08(1.80-45.88)$ & 0.008 & & \\
\hline Absence & 1.0 & & 1.0 & & & \\
\hline CD68 & $0.95(0.86-1.06)$ & 0.341 & & & & \\
\hline CD1a & $0.90(0.69-1.17)$ & 0.432 & & & & \\
\hline CD123 & $0.12(0.00-10.76)$ & 0.356 & & & & \\
\hline S100 & $1.02(0.88-1.19)$ & 0.799 & & & & \\
\hline CD208 & $1.41(0.66-3.03)$ & 0.380 & & & & \\
\hline CD83 & $1.66(0.18-14.97)$ & 0.652 & & & & \\
\hline \multicolumn{7}{|l|}{$\mathrm{ALN}^{-}$IR variables } \\
\hline CD4 & $0.98(0.96-1.01)$ & 0.217 & & & & \\
\hline CD8 & $1.08(1.02-1.14)$ & 0.010 & & & & \\
\hline CD57 & $1.86(0.45-7.77)$ & 0.395 & & & & \\
\hline F0XP3 & $1.00(1.00-1.00)$ & 0.285 & & & & \\
\hline CD21 & $0.96(0.81-1.14)$ & 0.611 & & & & \\
\hline CD68 & $1.11(1.02-1.21)$ & 0.012 & $1.24(1.05-1.47)$ & 0.013 & $1.27(1.10-1.47)$ & 0.001 \\
\hline
\end{tabular}


Breast Tumor Immunity in Axillary Nodes

Table 3 (continued)

\begin{tabular}{|c|c|c|c|c|c|c|}
\hline Variable & $\begin{array}{l}\text { Univariate } \\
\text { OR }(95 \% \mathrm{CI})\end{array}$ & $P$ value & $\begin{array}{l}\text { Multivariate } 1 \\
\text { OR }(95 \% \mathrm{CI})\end{array}$ & $P$ value & Multivariate 2 OR $(95 \% \mathrm{CI})$ & $P$ value \\
\hline CD1a & $1.01(0.90-1.14)$ & 0.856 & & & & \\
\hline CD123 & $0.67(0.50-0.90)$ & 0.007 & & & $0.44(0.27-0.72)$ & 0.001 \\
\hline S100 & $1.19(1.05-1.35)$ & 0.007 & & & $1.25(1.06-1.47)$ & 0.009 \\
\hline CD208 & $4.15(1.29-13.38)$ & 0.017 & & & & \\
\hline CD83 & $0.89(0.67-1.18)$ & 0.406 & & & & \\
\hline
\end{tabular}

ER, estrogen receptor; FOXP3, forkhead box P3; HER, human epidermal growth factor receptor; IR, immune response; LVI, lymphovascular invasion; OR, odds ratio; $\mathrm{PI}$, proliferation index; PNI, perineural invasion; $\mathrm{PR}$, progesterone receptor.

small subgroup of patients incorrectly classified as predicted-ALN ${ }^{-}$with a clear tendency to have similar clinical behavior to that of the diagnosed-ALN ${ }^{-}$. Moreover, as may be seen in the lower row, the great majority of diagnosed$\mathrm{ALN}^{-}$were correctly classified as predicted-ALN ${ }^{-}$, but there was also a small subgroup of patients incorrectly classified as predicted-ALN ${ }^{+}$with a clear tendency to have a similar clinical behavior to that of diagnosed-ALN ${ }^{+}$. These results strongly indicate that incorrect classification has a direct effect on the differentiation of patient outcome.

\section{Model Development and Adverse Effects of Incorrect Classification}

The classification of patients in each of the logistic regression models was based on the following formula:

$$
p=\frac{\exp (\alpha+\beta 1 \mathrm{X} 1+\ldots+\beta \mathrm{mXm}}{1+\exp (\alpha+\beta 1 \mathrm{X} 1+\ldots+\beta \mathrm{mXm}}
$$

The values of $P$ range from 0 to 1 , and the cutoff for belonging to the predicted-ALN ${ }^{-}$group was established as $\leq 0.5$ and $>0.5$ for the predicted-ALN ${ }^{+}$. The $\alpha$ and $\beta$ coefficients are estimated by the model, and the values of $X$ are provided for each of the patients for the variables included in each model. $\alpha$ is a constant, and the $\beta$ coefficients are calculated by the model. Each $\beta$ is calculated for each variable. For model 1, $\beta 1$ was the diameter of the tumor, $\beta 2$ was the LVI, $\beta 3$ was the intratumoral CD21, and $\beta 4$ was CD68 in $\mathrm{ALN}^{-}$. For model 2, $\beta 1$ was the diameter of the tumor, $\beta 2$ was CD68 in $\mathrm{ALN}^{-}, \beta 3$ was CD123 in $\mathrm{ALN}^{-}$, and $\beta 4$ was $\mathrm{S} 100$ in $\mathrm{ALN}^{-}$. In each model, these $\beta$ coefficients were constants for all the patients and each coefficient was multiplied by the value of the assigned variable (eg, $\beta 1$, the coefficient of the diameter of the tumor, is multiplied by $\mathrm{X} 1=10 \mathrm{~mm}$, the diameter of the tumor of one patient). The prediction errors of the logistic regression models arose because the values of the predictive variables $(\mathrm{X} 1, \mathrm{X} 2, \mathrm{X} 3, \mathrm{X} 4)$ in the incorrectly classified patients have values closer to those of patients of the opposite group. In this incorrect classification, the diagnosed$\mathrm{ALN}^{-}$were classified as predicted-ALN ${ }^{+}$because their probabilities arising from the formula were $>0.5$ instead of $\leq 0.5$. The same happened with the diagnosed-ALN ${ }^{+}$incorrectly classified as predicted-ALN ${ }^{-}$, as in the model the probabilities for these patients were $\leq 0.5$ instead of $>0.5$. So, the small subgroups of incorrectly classified patients arose from the tumor diameter (both models), LVI (model 1), and the concentration of the immune populations in the intratumoral area (model 1) and in the $\mathrm{ALN}^{-}$(both models). The differences in the variables of model 2 between correctly and incorrectly predicted patients are also partially confirmed by the comparison of the tumor diameter and the concentrations of immune populations in the $\mathrm{ALN}^{-}$(CD68, S100, and CD123) (Supplemental Table S1). In principle, the incorrect classification of patients should not have adverse effects other than the incorrect assignation of the patients to a particular group, as occurred with the first model. However, in the second model, this erroneous classification had an indirect effect on patients' clinical behavior, whereby a first small subgroup of diagnosed-ALN ${ }^{+}$incorrectly classified as predicted-ALN ${ }^{-}$had immune patterns in their $\mathrm{ALN}^{-}$more similar to those of the $\mathrm{ALN}^{-}$of the diagnosed-ALN ${ }^{-}$. This small subgroup of diagnosed-ALN ${ }^{+}$also showed clinical behavior similar to that of the diagnosed-ALN ${ }^{-}$group. The results also showed a second small subgroup of diagnosed$\mathrm{ALN}^{-}$incorrectly classified as predicted-ALN ${ }^{+}$, whose $\mathrm{ALN}^{-}$had immune patterns that were more similar to those of the $\mathrm{ALN}^{-}$of the diagnosed-ALN ${ }^{+}$group. This small subgroup of diagnosed-ALN ${ }^{-}$also showed similar clinical behavior to that of the diagnosed-ALN ${ }^{+}$. In model 1 , the incorrect classification of patients had no impact on their clinical behavior, but in model 2 , the incorrectly classified patients exhibited similar clinical behavior to those of the group in which they were incorrectly classified. The different outcome of the incorrectly classified patients could simply be a random effect of model 2, but the influence of the variables on the outcome of the patients was not evident in model 1. Moreover, in model 2, the change of outcomes in the two small subgroups of incorrectly classified patients indicated that the composition of the immune populations of the $\mathrm{ALN}^{-}$ could have an indirect impact on patient outcome, and leads us to rule out the possibility of being a random effect.

\section{Discussion}

To our knowledge, this is the first study using paraffinembedded samples to demonstrate that the patterns of immune populations of the $\mathrm{ALN}^{-}$differ between diagnosed- 

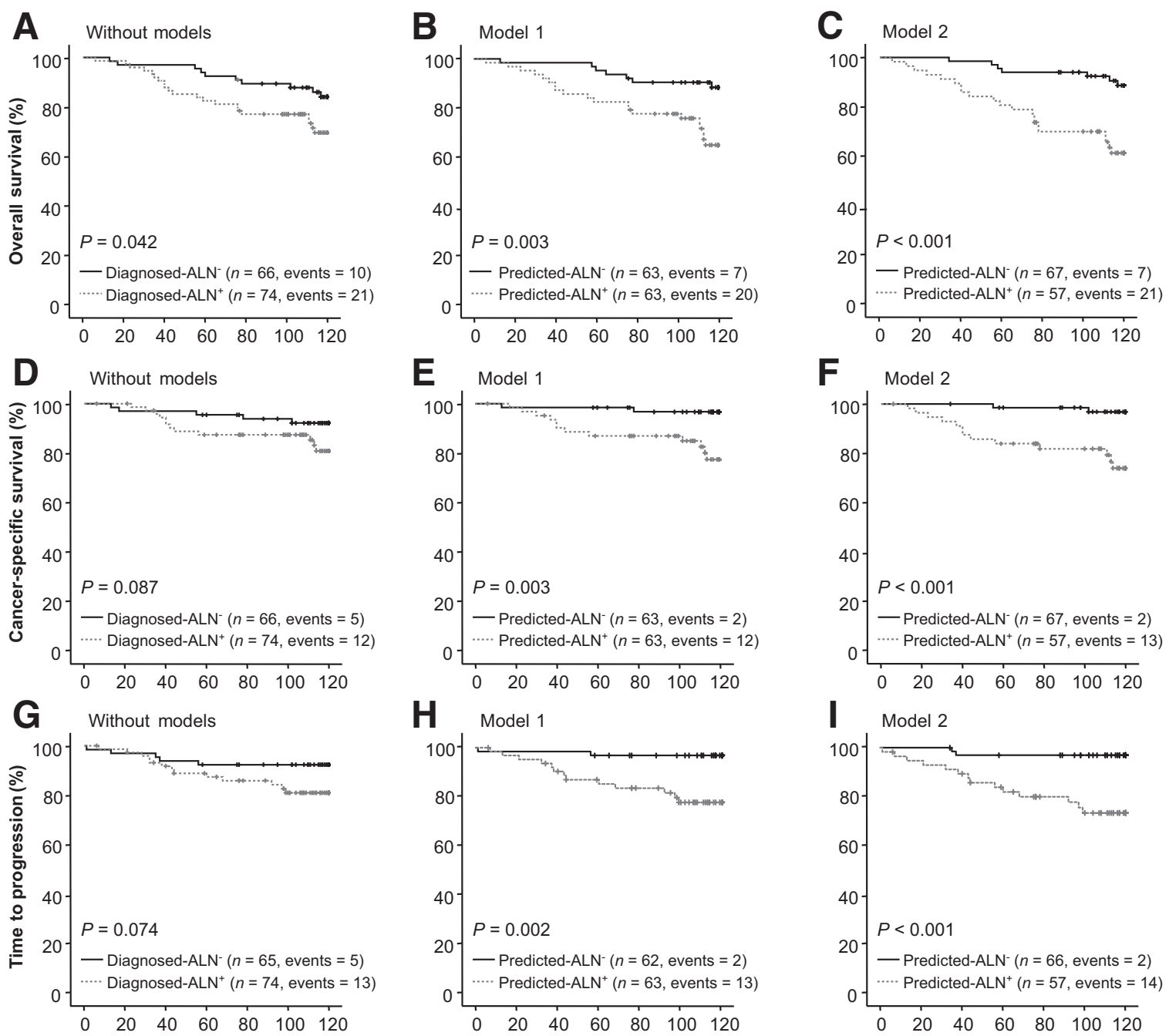

Figure 2 Kaplan-Meier survival curves for cases classified as diagnosed-ALN ${ }^{+}$and diagnosed-ALN ${ }^{-}$(without models) and classified as predicted-ALN ${ }^{+}$and predicted-ALN ${ }^{-}$according to the two models. Comparisons of the Kaplan-Meier curves illustrating overall survival (OS; A), cancer-specific survival (CSS; D), and time to progression (TTP; G) stratified between diagnosed-ALN ${ }^{+}$and diagnosed-ALN ${ }^{-}$patients. Comparisons of Kaplan-Meier curves illustrating 0S (B), CSS $(\mathbf{E})$, and TTP $(\mathbf{H})$ stratified between predicted-ALN ${ }^{+}$and predicted-ALN ${ }^{-}$for the model 1. Comparisons of Kaplan-Meier curves illustrating OS (C), CSS (F), and TTP (I) stratified between predicted-ALN ${ }^{+}$and predicted-ALN ${ }^{-}$for model 2.

$\mathrm{ALN}^{+}$and diagnosed-ALN ${ }^{-}$. The most significant finding herein is that some of these differences may have clinical implications for patient outcome.

It is well known that several clinical and pathologic factors used in current clinical practice are associated with the presence of $\mathrm{ALN}^{+} .^{32}$ Some recent studies have shown other histologic features linked to the presence of metastasis in ALN and to the prediction of developing distant metastasis (lymphocytic lobulitis and size and number or location of germinal centers). ${ }^{33}$ These new variables could be incorporated in the future to evaluate $\mathrm{BC}$ patient outcome. In this study, TMAs that, as previously mentioned, have been widely used in evaluations of tumor microenvironment biomarkers were used. However, a limitation of our study is that using TMAs is more difficult for studying other histologic or stromal variables, as noted by Grigoriadis et al. ${ }^{33}$ The use of well-established and new pathologic factors, together with the immune and stromal variables, could enable the more accurate prediction of $\mathrm{BC}$ patients. In our study, the main classic factors associated with the presence of $\mathrm{ALN}^{+}$at diagnosis in the univariate analyses coincide with those highlighted by other studies (ie, primary tumor size, histologic grade, LVI, and PNI) that are used in current clinical practice. $^{32,34}$ Our results suggest that LVI masks other variables associated with the circumstance of having $\mathrm{ALN}^{+}$at diagnosis. Therefore, the second model was developed, excluding the LVI, to determine which other variables were associated with $\mathrm{ALN}^{+}$.

The second model was worse at classifying patients, but had a special quality, whereby patients incorrectly predicted to be in a particular group had a similar clinical outcome to those actually in that group. These patients were incorrectly classified by model 2 because of the values of the predictive variables included in the model (tumor size and the immune 

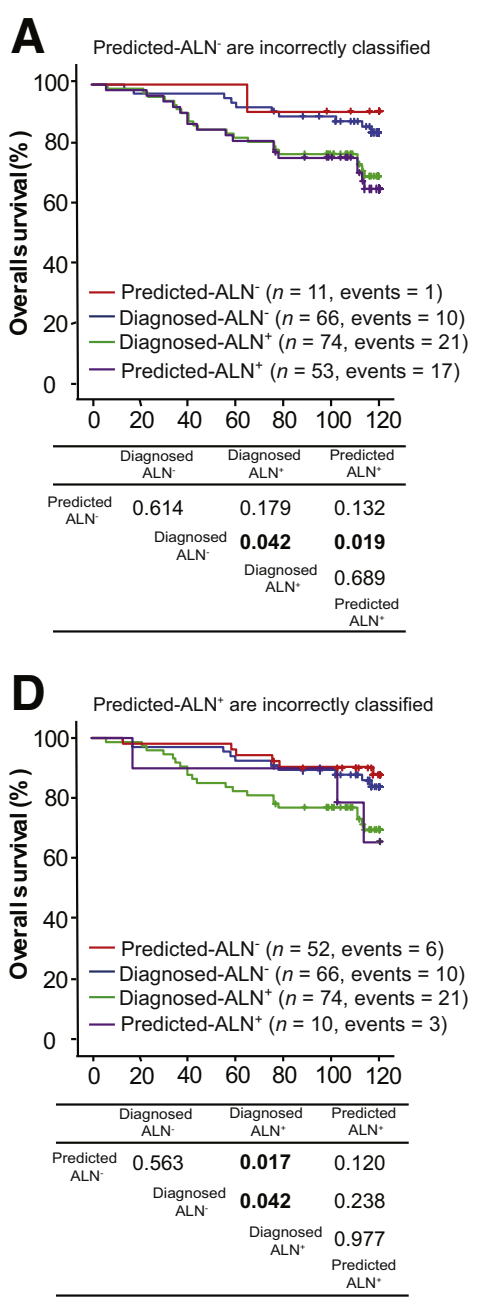
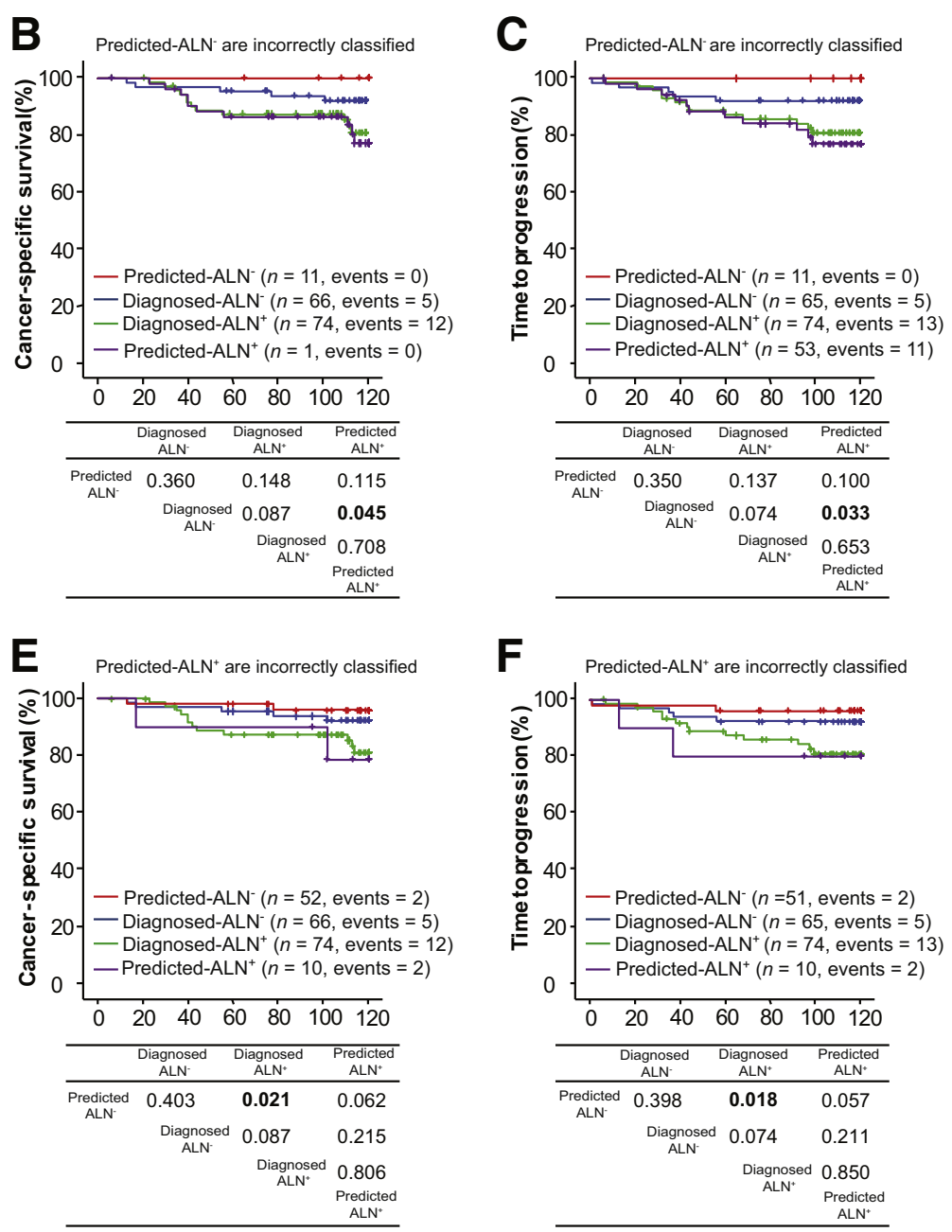

Figure 3 Kaplan-Meier curves for the survival times (overall survival, cancer-specific survival, and time to progression), compared using the log-rank test. A-C: Patients are those predicted by model 1 in the diagnosed-ALN ${ }^{+}$group: predicted-ALN ${ }^{+}$are correctly classified and predicted-ALN ${ }^{-}$are incorrectly classified. D-F: Patients are those predicted with model 1 in the diagnosed-ALN- group: predicted-ALN ${ }^{-}$are correctly classified and predicted-ALN ${ }^{+}$are incorrectly classified. Significant values of the log-rank-test appear in bold below each corresponding figure.

composition of their $\mathrm{ALN}^{-}$), and were more similar to those of the opposite group, highlighting how these variables could influence the survival and progression of BC patients. Thus, the small subgroup of diagnosed-ALN ${ }^{+}$that the model incorrectly classified as $\mathrm{ALN}^{-}$had a similar outcome to the diagnosed-ALN ${ }^{-}$patients. The same was the case for the small subgroup of diagnosed-ALN ${ }^{-}$that the model incorrectly classified as $\mathrm{ALN}^{+}$. These had a similar clinical outcome to the diagnosed-ALN ${ }^{+}$patients. One possible explanation for this variation is that the presence of the tumor in the ALN modifies the immune response in the neighboring $\mathrm{ALN}^{-}$, extending its ability to produce immune alterations beyond the tumor's physical location, in most cases. Nevertheless, a small proportion of patients (diagnosed-ALN ${ }^{+}$who were incorrectly classified as predicted$\mathrm{ALN}^{-}$by model 2) can maintain their immune patterns in the $\mathrm{ALN}^{-}$because the tumor in the $\mathrm{ALN}^{+}$is not able to modify them. This maintenance might also be responsible for the better outcome of these patients.
However, there needs to be an explanation for what happened in the small group of diagnosed-ALN ${ }^{-}$patients who were incorrectly classified as predicted-ALN ${ }^{+}(6.8 \%)$, and who had a similar clinical outcome to those of the group into which they were erroneously classified (diagnosed$\mathrm{ALN}^{+}$). One possible explanation is the presence of occult metastasis that can alter the immune patterns of the $\mathrm{ALN}^{-}$in this group, thereby leading to the incorrect classification in model 2. Occult metastasis has been estimated to occur in up to $20 \%$ of patients, ${ }^{4}$ and although the results published so far are not conclusive, it seems that their presence may be associated with poorer disease-free survival and $\mathrm{OS}^{35}$ as observed in this small group of incorrectly predicted patients. Another possible explanation emerges from the results of Zuckerman et al, ${ }^{11}$ who showed that the modifications/alterations of the gene expression profiles of immune cells in diagnosed-ALN ${ }^{+}$and diagnosed-ALN ${ }^{-}$occur not only in the primary tumor or the ALN, but also in peripheral blood. Under these conditions, the differences in this group of 

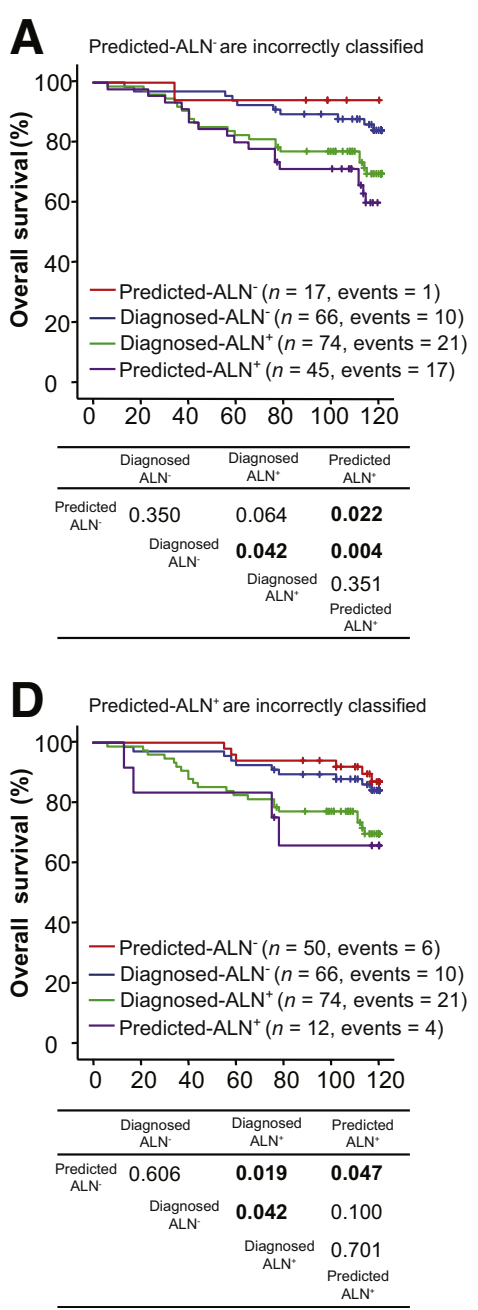
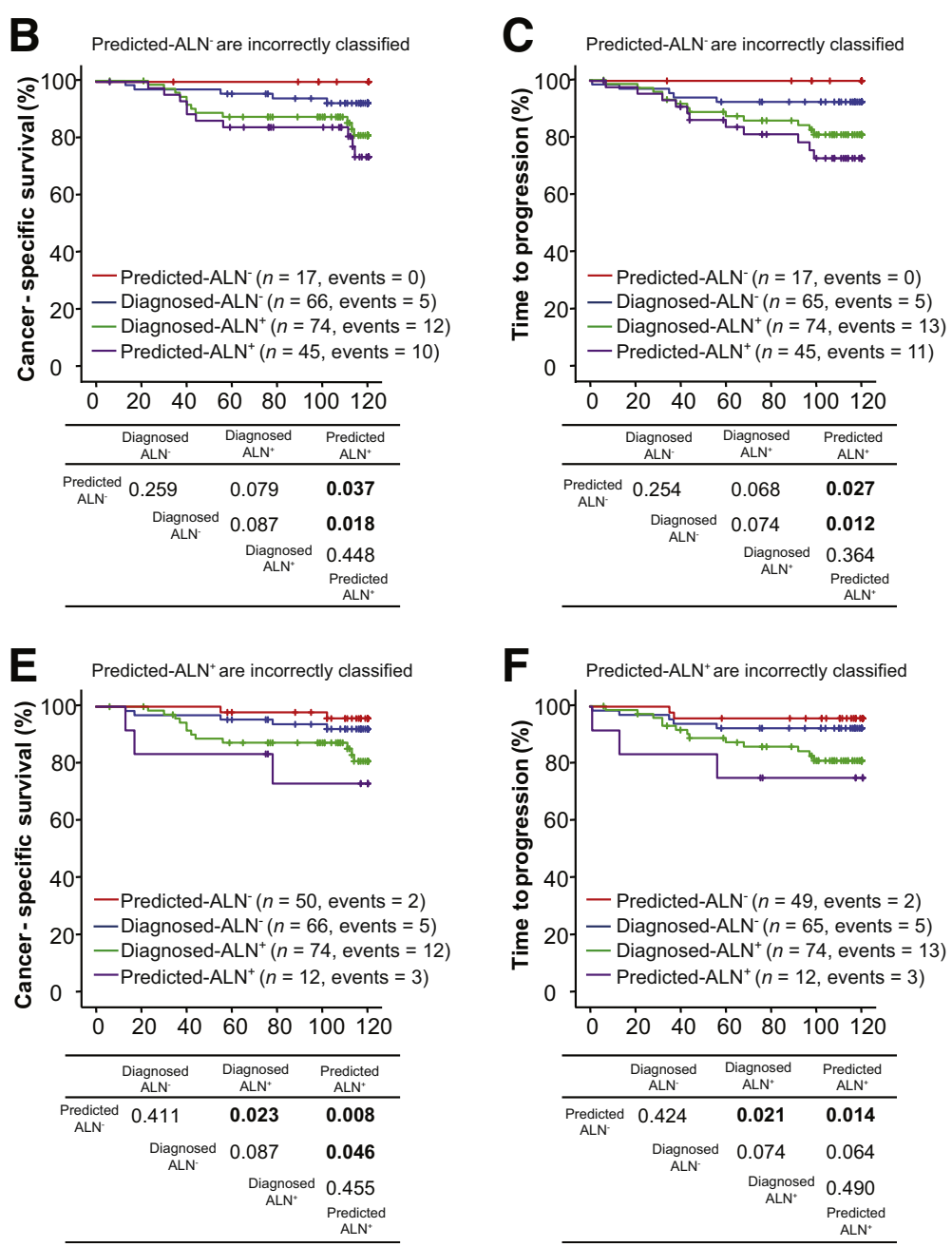

Figure 4 Kaplan-Meier curves for the survival times (overall survival, cancer-specific survival, and time to progression), compared using the log-rank test. A-C: Patients are those predicted by model 2 in the diagnosed-ALN ${ }^{+}$group: predicted-ALN ${ }^{+}$are correctly classified and predicted-ALN ${ }^{-}$are incorrectly classified. D-F: Patients are those predicted by model 2 in the diagnosed-ALN ${ }^{-}$group: predicted-ALN ${ }^{-}$are correctly classified and predicted-ALN ${ }^{+}$are incorrectly classified. Significant values of the log-rank-test appear in bold below each corresponding figure.

diagnosed- $\mathrm{ALN}^{-}$that are classified as predicted-ALN ${ }^{+}$could be the consequence of cancer cells that have escaped into the blood being detected by the systemic immune cells that can, in turn, alter the ALN immune response pattern. Something like what Zuckerman et al ${ }^{11}$ described could also occur to the $\mathrm{ALN}^{-}$of diagnosed-ALN ${ }^{+}$that have still not been invaded because they have a different immune response pattern to that of the $\mathrm{ALN}^{-}$of diagnosed-ALN ${ }^{-}$. In this latter case, these changes may not be produced directly by a neighboring $\mathrm{ALN}^{+}$, but rather by the systemic immune cells. This implies that the detection of this small proportion of more immunocompetent diagnosed-ALN ${ }^{+}$in which their $\mathrm{ALN}^{-}$remains intact, like the $\mathrm{ALN}^{-}$of the $\mathrm{ALN}^{-}$, could be of great importance for their follow-up.

With respect to the specific immune populations of the ALN, the results show that $\mathrm{CD} 68^{+}$macrophages are one of the immune populations that proved to be relevant at the level of $\mathrm{ALN}^{-}$. In $\mathrm{BC}$, a high density of tumor-associated macrophages (TAMs) is correlated with poor prognosis. ${ }^{36}$
However, M1 macrophages are capable of activating the type I T-lymphocyte response; they are also cytotoxic to neoplastic cells and are favorable antigen-presenting cells. By contrast, M2 macrophages have lower antigen-presenting capacities, can promote angiogenesis, and are capable of inhibiting the immune response of M1 macrophages. ${ }^{37}$ For these reasons, M2 macrophages are believed to be detrimental in cancer. ${ }^{38}$ In our case, the macrophages are not considered to be genuine TAMs because they do not infiltrate the primary tumor; instead, their presence in the peripheral ALN leads to them being considered a peripheral TAM. However, the increase in the concentration of peripheral TAMs in the $\mathrm{ALN}^{-}$seems to be associated with the presence of $\mathrm{ALN}^{+}$at diagnosis. That the presence of these peripheral TAMs seems to be associated with poor prognosis implies that they could also be polarized (M2 phenotype), as are the TAMs present in many other types of cancer. ${ }^{39,40}$ A better identification of the types and function of peripheral TAM populations present in the $\mathrm{ALN}^{-}$should be investigated in the future. 
The other two populations of immune cells present in the $\mathrm{ALN}^{-}$that were found to be associated with the presence of $\mathrm{ALN}^{+}$at diagnosis were both DCs, specifically plasmacytoid DC (CD123) and interdigitant DC (S100). DCs are key regulators of cancer immunity because they can process and present tumor-associated antigens, especially in the draining lymph nodes, ${ }^{41,42}$ where $\mathrm{T}$ cells are primed and activated against specific cancer antigens. ${ }^{43}$ Nevertheless, this cycle is not always completed because of the level of local and systemic immune suppression. ${ }^{42,43}$ Generally, tumorinfiltrating CD123 DCs are associated with bad prognosis as they tend to be tolerogenic and promote an immunosuppressive microenvironment that activates T-regulatory cells in BC patients and is correlated with a worse outcome. ${ }^{44,45}$ However, it has been demonstrated in melanoma that CD123 DCs can promote the immunogenic response against the tumor if they are properly stimulated. ${ }^{46}$ To the best of our knowledge, the only study of BC that has evaluated CD123 in the ALN found no differences between $\mathrm{ALN}^{+}$and $\mathrm{ALN}^{-}$. . Nevertheless, as occurs with macrophages, these CD123 DCs do not infiltrate the tumor; their role in tumor development remains unclear. In this study, it seems that an increase in these immune populations is associated with being a factor protecting against having $\mathrm{ALN}^{+}$.

Finally, interdigitating DCs (S100) are bone marrow-derived DCs located in the T-cell domains of various humans tissues. ${ }^{47}$ The results are similar to those seen in macrophages, whereby an increase in S100 DC concentration in the $\mathrm{ALN}^{-}$is associated with having $\mathrm{ALN}^{+}$ at diagnosis. No studies have examined whether there are differences in the $\mathrm{ALN}^{-} \mathrm{S} 100$ between diagnosed-ALN ${ }^{+}$ and diagnosed-ALN ${ }^{-}$patients, and we are not aware of any that have evaluated the differences between $\mathrm{ALN}^{+}$and $\mathrm{ALN}^{-}$in $\mathrm{BC}$.

Considering all these results together, the three immune populations identified by the second model are associated, in this and other studies, with a particular response to cancer, either through increasing the immune response to tumoral cells or by inducing a tolerogenic environment ${ }^{42,48}$ that then affects patient prognosis. ${ }^{7}$

In conclusion, this study features two key results related to some immune components of the $\mathrm{ALN}^{-}$. The first is that some immune cell populations of the $\mathrm{ALN}^{-}$are more strongly associated with having metastasis in the ALN at diagnosis than some classic pathologic factors that are also associated with $\mathrm{ALN}^{+}$. This is evident from the second multivariate model as the histologic grade, PNI, and proliferation index were excluded from the model when combined with these immune populations. Although immune cell populations may not be more important than the pathologic factors for predicting the outcome of patients, they are complementary. The second result is that these immune cell populations of the $\mathrm{ALN}^{-}$may be associated with patient outcome in the small subgroups of incorrectly predicted patients. These results indicate that patient outcome depends not only on whether a patient has $\mathrm{ALN}^{+}$but also on the type of immune population of their $\mathrm{ALN}^{-}$. If diagnosed-ALN ${ }^{+}$have $\mathrm{ALN}^{-}$patterns in their immune populations that are more similar to those of diagnosed-ALN ${ }^{-}$, then they tend also to exhibit clinical behavior similar to that of the group of diagnosed-ALN ${ }^{-}$. This also happens in the reciprocal situation, whereby the diagnosed-ALN ${ }^{-}$whose $\mathrm{ALN}^{-}$patterns in the immune populations are more similar to those of diagnosed-ALN ${ }^{+}$patients will tend also to show similar clinical behavior to the diagnosed-ALN ${ }^{+}$group.

Our current results suggest a new direction that could be pursued in the study of the immune response in $\mathrm{BC}$ patients, not only in the primary tumor and in the $\mathrm{ALN}^{+}$, as other studies have addressed, but also in the $\mathrm{ALN}^{-}$. Although great advances are expected from the new immunotherapeutic treatments, preclinical investigation remains necessary to understand why clinical trials fail, or simply to know what is happening in cancer development. ${ }^{49}$ The study of the immune response in unaffected lymph nodes could be one of the key factors. This approach to the study of immune populations in the regional negative lymph nodes could also be adopted to investigate other cancers, such as colorectal cancer and melanoma, in which the immune status of negative lymph nodes may also be an important factor related to the clinical outcome of patients.

\section{Acknowledgments}

We thank Barbara Tomás, Vanesa Gestí, María del Mar Barbera, Anna Curto, Noelia Burgues, Ainhoa Montserrat, Eduard Nolla, Maria Fortuny, Sandra Bages, Mireia Sueca, and Marc Iniesta for skillful technical assistance; and Anna Carot and Rosa Cabrera for excellent secretarial work.

\section{Supplemental Data}

Supplemental material for this article can be found at https://doi.org/10.1016/j.ajpath.2019.11.002.

\section{References}

1. Ferlay J, Soerjomataram I, Dikshit R, Eser S, Mathers C, Rebelo M, Parkin DM, Forman D, Bray F: Cancer incidence and mortality worldwide: sources, methods and major patterns in GLOBOCAN 2012. Int J Cancer 2015, 136:E359-E386

2. Siegel RL, Miller KD, Jemal A: Cancer statistics, 2017. CA Cancer J Clin 2017, 67:7-30

3. Colzani E, Johansson AL, Liljegren A, Foukakis T, Clements M, Adolfsson J, Hall P, Czene K: Time-dependent risk of developing distant metastasis in breast cancer patients according to treatment, age and tumour characteristics. Br J Cancer 2014, 110:1378-1384

4. Bernet Vegue L, Cano Munoz R, Pinero Madrona A: Breast cancer sentinel lymph node and axillary lymphadenectomy: new tools for new challenges. Expert Rev Mol Diagn 2012, 12:147-158

5. Pages F, Galon J, Dieu-Nosjean MC, Tartour E, Sautes-Fridman C, Fridman WH: Immune infiltration in human tumors: a prognostic factor that should not be ignored. Oncogene 2010, 29:1093-1102 
6. Fridman WH, Galon J, Pages F, Tartour E, Sautes-Fridman C, Kroemer G: Prognostic and predictive impact of intra- and peritumoral immune infiltrates. Cancer Res 2011, 71:5601-5605

7. de la Cruz-Merino L, Barco-Sanchez A, Henao Carrasco F, Nogales Fernandez E, Vallejo Benitez A, Brugal Molina J, Martinez Peinado A, Grueso Lopez A, Ruiz Borrego M, Codes Manuel de Villena M, Sanchez-Margalet V, Nieto-Garcia A, Alba Conejo E, Casares Lagar N, Ibanez Martinez J: New insights into the role of the immune microenvironment in breast carcinoma. Clin Dev Immunol 2013, 2013:785317

8. Poindexter NJ, Sahin A, Hunt KK, Grimm EA: Analysis of dendritic cells in tumor-free and tumor-containing sentinel lymph nodes from patients with breast cancer. Breast Cancer Res 2004, 6:R408-R415

9. Mansfield AS, Heikkila P, von Smitten K, Vakkila J, Leidenius M: Metastasis to sentinel lymph nodes in breast cancer is associated with maturation arrest of dendritic cells and poor co-localization of dendritic cells and CD8 + T cells. Virchows Arch 2011, 459:391-398

10. Kim R, Emi M, Tanabe K, Arihiro K: Immunobiology of the sentinel lymph node and its potential role for antitumour immunity. Lancet Oncol 2006, 7:1006-1016

11. Zuckerman NS, Yu H, Simons DL, Bhattacharya N, CarcamoCavazos V, Yan N, Dirbas FM, Johnson DL, Schwartz EJ, Lee PP: Altered local and systemic immune profiles underlie lymph node metastasis in breast cancer patients. Int J Cancer 2013, 132: $2537-2547$

12. Matsuura K, Yamaguchi Y, Ueno H, Osaki A, Arihiro K, Toge T: Maturation of dendritic cells and T-cell responses in sentinel lymph nodes from patients with breast carcinoma. Cancer 2006, 106: $1227-1236$

13. Callau C, Lejeune M, Korzynska A, Garcia M, Bueno G, Bosch R, Jaen J, Orero G, Salvado T, Lopez C: Evaluation of cytokeratin-19 in breast cancer tissue samples: a comparison of automatic and manual evaluations of scanned tissue microarray cylinders. Biomed Eng Online 2015, 14 Suppl 2:S2

14. Pinder SE, Brown JP, Gillett C, Purdie CA, Speirs V, Thompson AM, Shaaban AM: The manufacture and assessment of tissue microarrays: suggestions and criteria for analysis, with breast cancer as an example. J Clin Pathol 2013, 66:169-177

15. Pelekanou V, Villarroel-Espindola F, Schalper KA, Pusztai L, Rimm DL: CD68, CD163, and matrix metalloproteinase 9 (MMP-9) co-localization in breast tumor microenvironment predicts survival differently in ER-positive and -negative cancers. Breast Cancer Res 2018, 20:154

16. Solinas C, Garaud S, De Silva P, Boisson A, Van den Eynden G, de Wind A, Risso P, Rodrigues Vitoria J, Richard F, Migliori E, Noel G, Duvillier H, Craciun L, Veys I, Awada A, Detours V, Larsimont D, Piccart-Gebhart M, Willard-Gallo K: Immune checkpoint molecules on tumor-infiltrating lymphocytes and their association with tertiary lymphoid structures in human breast cancer. Front Immunol 2017, 8: 1412

17. Hamilton PW, Bankhead P, Wang Y, Hutchinson R, Kieran D, McArt DG, James J, Salto-Tellez M: Digital pathology and image analysis in tissue biomarker research. Methods 2014, 70:59-73

18. de Melo Gagliato D, Cortes J, Curigliano G, Loi S, Denkert C, PerezGarcia J, Holgado E: Tumor-infiltrating lymphocytes in breast cancer and implications for clinical practice. Biochim Biophys Acta Rev Cancer 2017, 1868:527-537

19. Zhao X, Qu J, Sun Y, Wang J, Liu X, Wang F, Zhang H, Wang W, Ma X, Gao X, Zhang S: Prognostic significance of tumor-associated macrophages in breast cancer: a meta-analysis of the literature. Oncotarget 2017, 8:30576-30586

20. Stovgaard ES, Nielsen D, Hogdall E, Balslev E: Triple negative breast cancer - prognostic role of immune-related factors: a systematic review. Acta Oncol 2018, 57:74-82

21. Bueno G, Fernandez-Carrobles MM, Deniz O, Garcia-Rojo M: New trends of emerging technologies in digital pathology. Pathobiology 2016, 83:61-69
22. Fernandez-Carrobles Mdel M, Bueno G, Deniz O, Salido J, GarciaRojo M: Automatic handling of tissue microarray cores in highdimensional microscopy images. IEEE J Biomed Health Inform 2014, 18:999-1007

23. Roszkowiak L, Lopez C: PATMA: parser of archival tissue microarray. Peer J 2016, 4:e2741

24. Gandomkar Z, Brennan PC, Mello-Thoms C: Computer-based image analysis in breast pathology. J Pathol Inform 2016, 7:43

25. Lopez C, Lejeune M, Bosch R, Korzynska A, Garcia-Rojo M, Salvado MT, Alvaro T, Callau C, Roso A, Jaen J: Digital image analysis in breast cancer: an example of an automated methodology and the effects of image compression. Stud Health Technol Inform 2012, 179:155-171

26. Lloyd MC, Johnson JO, Kasprzak A, Bui MM: Image analysis of the tumor microenvironment. Adv Exp Med Biol 2016, 936:1-10

27. Korzynska A, Neuman U, Lopez C, Lejeune M, Bosch R: The method of immunohistochemical images standardization. Edited by Choras RS. In Image Processing and Communications Challenges 2. Berlin, Heidelberg: Springer, 2010. pp. 213-221

28. Korzynska A, Roszkowiak L, Jakub Z, Lejeune M, Orero G, Bosch R, Lopez C: The METINUS Plus method for nuclei quantification in tissue microarrays of breast cancer and axillary node tissue section. Biomed Signal Process Control 2017, 32:1-9

29. Peduzzi P, Concato J, Kemper E, Holford TR, Feinstein AR: A simulation study of the number of events per variable in logistic regression analysis. J Clin Epidemiol 1996, 49:1373-1379

30. Vittinghoff $\mathrm{E}, \mathrm{McCulloch} \mathrm{CE}$ : Relaxing the rule of ten events per variable in logistic and Cox regression. Am J Epidemiol 2007, 165: 710-718

31. DeLong ER, DeLong DM, Clarke-Pearson DL: Comparing the areas under two or more correlated receiver operating characteristic curves: a nonparametric approach. Biometrics 1988, 44:837-845

32. Patani NR, Dwek MV, Douek M: Predictors of axillary lymph node metastasis in breast cancer: a systematic review. Eur J Surg Oncol 2007, 33:409-419

33. Grigoriadis A, Gazinska P, Pai T, Irhsad S, Wu Y, Millis R, Naidoo K, Owen J, Gillett CE, Tutt A, Coolen AC, Pinder SE: Histological scoring of immune and stromal features in breast and axillary lymph nodes is prognostic for distant metastasis in lymph node-positive breast cancers. J Pathol Clin Res 2018, 4:39-54

34. Tseng HS, Chen LS, Kuo SJ, Chen ST, Wang YF, Chen DR: Tumor characteristics of breast cancer in predicting axillary lymph node metastasis. Med Sci Monit 2014, 20:1155-1161

35. de Boer M, van Dijck JA, Bult P, Borm GF, Tjan-Heijnen VC: Breast cancer prognosis and occult lymph node metastases, isolated tumor cells, and micrometastases. J Natl Cancer Inst 2010, 102:410-425

36. Choi J, Gyamfi J, Jang H, Koo JS: The role of tumor-associated macrophage in breast cancer biology. Histol Histopathol 2018, 32: 133-145

37. Solinas G, Germano G, Mantovani A, Allavena P: Tumor-associated macrophages (TAM) as major players of the cancer-related inflammation. J Leukoc Biol 2009, 86:1065-1073

38. Mantovani A, Marchesi F, Malesci A, Laghi L, Allavena P: Tumourassociated macrophages as treatment targets in oncology. Nat Rev Clin Oncol 2017, 14:399-416

39. Alvaro T, Lejeune M, Camacho FI, Salvado MT, Sanchez L, Garcia JF, Lopez C, Jaen J, Bosch R, Pons LE, Bellas C, Piris MA: The presence of STAT1-positive tumor-associated macrophages and their relation to outcome in patients with follicular lymphoma. Haematologica 2006, 91:1605-1612

40. Yuan R, Li S, Geng H, Wang X, Guan Q, Li X, Ren C, Yuan X: Reversing the polarization of tumor-associated macrophages inhibits tumor metastasis. Int Immunopharmacol 2017, 49:30-37

41. da Cunha A, Michelin MA, Murta EF: Pattern response of dendritic cells in the tumor microenvironment and breast cancer. World J Clin Oncol 2014, 5:495-502

42. Gardner A, Ruffell B: Dendritic cells and cancer immunity. Trends Immunol 2016, 37:855-865 
43. Chen DS, Mellman I: Oncology meets immunology: the cancerimmunity cycle. Immunity 2013, 39:1-10

44. Swiecki M, Colonna M: The multifaceted biology of plasmacytoid dendritic cells. Nat Rev Immunol 2015, 15:471-485

45. Treilleux I, Blay JY, Bendriss-Vermare N, Ray-Coquard I, Bachelot T, Guastalla JP, Bremond A, Goddard S, Pin JJ, Barthelemy-Dubois C, Lebecque S: Dendritic cell infiltration and prognosis of early stage breast cancer. Clin Cancer Res 2004, 10:7466-7474

46. Tel J, Aarntzen EH, Baba T, Schreibelt G, Schulte BM, BenitezRibas D, Boerman OC, Croockewit S, Oyen WJ, van Rossum M,
Winkels G, Coulie PG, Punt CJ, Figdor CG, de Vries IJ: Natural human plasmacytoid dendritic cells induce antigen-specific T-cell responses in melanoma patients. Cancer Res 2013, 73:1063-1075

47. Bodey B, Siegel SE, Kaiser HE: Antigen presentation by dendritic cells and their significance in antineoplastic immunotherapy. In Vivo 2004, 18:81-100

48. Chen DS, Mellman I: Elements of cancer immunity and the cancerimmune set point. Nature 2017, 541:321-330

49. The Lancet Oncology: Calling time on the immunotherapy gold rush. Lancet Oncol 2017, 18:981 